\title{
Magnetically Confined Wind Shocks in X-rays \\ - a Review
}

\author{
Asif ud-Doula*
}

Penn State Worthington Scranton, 120 Ridge View Drive, Dunmore, PA 18512, USA

Yaël Nazé ${ }^{1, * *}$

Institut d'Astrophysique et de Géophysique, Université de Liège, Quartier Agora, Allée du 6 Août 19c, Bat. B5C, B4000-Liège, Belgium

\begin{abstract}
A subset $(\sim 10 \%)$ of massive stars present strong, globally ordered (mostly dipolar) magnetic fields. The trapping and channeling of their stellar winds in closed magnetic loops leads to magnetically confined wind shocks (MCWS), with pre-shock flow speeds that are some fraction of the wind terminal speed. These shocks generate hot plasma, a source of $\mathrm{X}$-rays. In the last decade, several developments took place, notably the determination of the hot plasma properties for a large sample of objects using $X M M$ and Chandra, as well as fully self-consistent MHD modelling and the identification of shock retreat effects in weak winds. Despite a few exceptions, the combination of magnetic confinement, shock retreat and rotation effects seems to be able to account for X-ray emission in massive OB stars. Here we review these new observational and theoretical aspects of this X-ray emission and envisage some perspectives for the next generation of X-ray observatories.
\end{abstract}

Keywords: X-rays, MHD, Massive Stars

\section{Introduction}

Hot luminous, massive stars of spectral type $\mathrm{O}$ and $\mathrm{B}$ are prominent sources of X-rays which can originate from three distinct sources: shocks in their high-speed radiatively driven stellar winds, wind-wind collisions in binary systems and magnetically confined wind shocks.

In single, non-magnetic $\mathrm{O}$ stars, the intrinsic instability of wind driving by line-scattering leads to embedded wind shocks that are thought to be the source of their relatively soft $(\sim 0.5 \mathrm{keV})$ X-ray spectrum, with a total X-ray luminosity that scales with stellar bolometric luminosity, $L_{\mathrm{x}} \sim 10^{-7} \times L_{\text {bol }}$ (Chlebowski, 1989; Berghoefer et al., 1997; Nazé et al., 2011). In massive binary systems the collision of the two stellar winds at up to the wind terminal speeds can lead to even higher $L_{\mathrm{x}}$, generally with a significantly harder (up to $10 \mathrm{keV}$ ) spectrum (for a review, see Rauw \& Nazé, this volume).

Here we discuss a third source of $\mathrm{X}$-rays from $\mathrm{OB}$ winds, namely those observed from the subset $(\sim 10 \%)$ of massive stars with strong, globally ordered (often significantly dipolar) magnetic fields (Petit et al., 2013); in this case, the trapping and channeling of the stellar wind in closed

\footnotetext{
* Corresponding author - theory

** Corresponding author - observations

Email addresses: asif@psu.edu (Asif ud-Doula),

naze@astro.ulg.ac.be (Yaël Nazé)

${ }^{1}$ Research Associate FRS-FNRS
}

magnetic loops leads to magnetically confined wind shocks (MCWS) (Babel and Montmerle, 1997a,b), with pre-shock flow speeds that are some fraction of the wind terminal speed, resulting in intermediate energies for the shocks and associated X-rays $(\sim 2 \mathrm{keV})$. A prototypical example is provided by the magnetic O-type star $\theta^{1}$ Ori $\mathrm{C}$, which shows moderately hard X-ray emission with a rotational phase variation that matches well the expectations of the MCWS paradigm (Gagné et al., 2005a).

Here, we discuss theoretical aspects of magnetic confinement that determine the extent of the influence of the field over the wind. We, then, describe an effect called 'shock retreat', which can moderate the strength of the Xrays, or even quench it altogether in extremely low mass loss rate stars, and the effects of rotation. In $\S 3$, we describe current results in the X-ray observations of earlytype magnetic stars, while $\S 4$ briefly discusses future outlooks of MCWS in X-ray astronomy.

\section{Theoretical Perspective}

To explain X-ray emission from the Ap/Bp star IQ Aur Babel and Montmerle (1997a) introduced the MCWS model. In their approach, they effectively prescribed a fixed magnetic field geometry to channel the wind outflow (see also Shore and Brown 1990). For large magnetic loops, wind material from opposite footpoints is accelerated to a substantial fraction of the wind terminal speed (i.e., $\geq 1000$ 
$\mathrm{km} \mathrm{s}^{-1}$ ) before the channeling toward the loop tops forces a collision with very strong shocks, thereby heating the gas to temperatures $\left(10^{7}-10^{8} \mathrm{~K}\right)$ that are high enough to emit hard (few keV) X-rays. This star has a quite strong field $(\sim 4 \mathrm{kG})$ and a rather weak wind, with an estimated mass loss rate of about $\sim 10^{-10} M_{\odot} \mathrm{yr}^{-1}$, and thus indeed could be reasonably modeled within the framework of prescribed magnetic field geometry. However, the actual $\mathrm{X}$-ray emission from the star, which is predominantly soft, is further influenced by an effect called 'shock retreat' that we describe below. Later, Babel and Montmerle (1997b) applied this model to explain the periodic variation of X-ray emission of the O7 star $\theta^{1}$ Ori C, which has a lower magnetic field $(\sim 1100 \mathrm{G})$ and significantly stronger wind (mass-loss rate $\sim 10^{-7} M_{\odot}$ $\mathrm{yr}^{-1}$ ), raising now the possibility that the wind itself could influence the field geometry in a way that is not considered in the simple fixed-field approach.

\subsection{Magnetic Confinement}

In an interplay between magnetic field and stellar wind, the dominance of the field is determined by how strong it is relative to the wind. To understand the competition between these two, ud-Doula and Owocki (2002) defined a characteristic parameter for the relative effectiveness of the magnetic fields in confining and/or channeling the wind outflow. Specifically, consider the ratio between the energy densities of field vs. flow,

$$
\begin{aligned}
\eta(r, \theta) & \equiv \frac{B^{2} / 8 \pi}{\rho v^{2} / 2} \approx \frac{B^{2} r^{2}}{\dot{M} v(r)} \\
& =\left[\frac{B_{*}^{2}(\theta) R_{*}{ }^{2}}{\dot{M} v_{\infty}}\right]\left[\frac{\left(r / R_{*}\right)^{-2 n}}{1-R_{*} / r}\right],
\end{aligned}
$$

where the latitudinal variation of the surface field has the dipole form given by $B_{*}^{2}(\theta)=B_{o}^{2}\left(\cos ^{2} \theta+\sin ^{2} \theta / 4\right)$. In general, a magnetically channeled outflow will have a complex flow geometry, but for convenience, the second equality in eqn. (1) simply characterizes the wind strength in terms of a spherically symmetric mass loss rate $\dot{M}=4 \pi r^{2} \rho v$. The third equality likewise characterizes the radial variation of outflow velocity in terms of the phenomenological velocity law $v(r)=v_{\infty}\left(1-R_{*} / r\right)^{\beta}$, with $v_{\infty}$ the wind terminal speed and $\beta=1$; this equation furthermore models the magnetic field strength decline as a power-law in radius, $B(r)=B_{*}\left(R_{*} / r\right)^{(n+1)}$, where, e.g., for a dipole $n=2$.

With the spatial variations of this energy ratio thus isolated within the right square bracket, we see that the left square bracket represents a dimensionless constant that characterizes the overall relative strength of field vs. wind. Evaluating this in the region of the magnetic equator $(\theta=$ $90^{\circ}$ ), where the tendency toward a radial wind outflow is in most direct competition with the tendency for a horizontal orientation of the field, one can thus define an equatorial 'wind magnetic confinement parameter',

$$
\eta_{*} \equiv \frac{B_{*}^{2}\left(90^{\circ}\right) R_{*}{ }^{2}}{\dot{M} v_{\infty}}=0.4 \frac{B_{100}^{2} R_{12}^{2}}{\dot{M}_{-6} v_{8}} .
$$

where $\dot{M}_{-6} \equiv \dot{M} /\left(10^{-6} M_{\odot} / \mathrm{yr}\right), B_{100} \equiv B_{o} /(100 \mathrm{G})$, $R_{12} \equiv R_{*} /\left(10^{12} \mathrm{~cm}\right)$, and $v_{8} \equiv v_{\infty} /\left(10^{8} \mathrm{~cm} / \mathrm{s}\right)$. In order to have any confinement, $\eta_{*} \geq 1$. As these stellar and wind parameters are scaled to typical values for an OB supergiant, e.g. $\zeta$ Pup, the last equality in eqn. (2) immediately suggests that for such winds, significant magnetic confinement or channeling should require fields of order few hundred G. By contrast, in the case of the sun, the much weaker mass loss $\left(\dot{M}_{\odot} \sim 10^{-14} M_{\odot} / \mathrm{yr}\right)$ means that even a much weaker global field $\left(B_{o} \sim 1 \mathrm{G}\right)$ is sufficient to yield $\eta_{*} \simeq 40$, implying a substantial magnetic confinement of the solar coronal expansion. But in Bp stars the magnetic field strength can be of order $\mathrm{kG}$ with $\dot{M}_{\odot} \sim 10^{-10} M_{\odot} /$ yr leading $\eta_{*} \leq 10^{6}$. Thus, the confinement in Bp stars is very extreme.

It should be emphasized that $\dot{M}$ used in the above formalism is a value obtained for a spherically symmetric non-magnetic wind as the magnetic field may significantly influence the predicted circumstellar density and velocity structure.

\subsubsection{Alfúen Radius}

The extent of the effectiveness of magnetic confinement is set by the Alfvén radius, $R_{A}$, where flow and Alfvén velocities are equal. This will also determine the extent of the largest loops and thus the highest shock velocities affecting the hardness of X-ray emission. This radius can be derived from eqn. (1) where the second square bracket factor shows the overall radial variation; $n$ is the power-law exponent for radial decline of the assumed stellar field, e.g. $n=2$ for a pure dipole, and with $v(r)=v_{\infty}\left(1-R_{*} / r\right)^{\beta}$ where $\beta$ is the velocity-law index (typically $\beta \approx 1$ ). For a star with a non-zero field, we have $\eta_{*}>0$, and so given the vanishing of the flow speed at the atmospheric wind base, this energy ratio always starts as a large number near the stellar surface, $\eta\left(r \rightarrow R_{*}\right) \rightarrow \infty$. But from there outward it declines quite steeply, asymptotically as $r^{-4}$ for a dipole, crossing unity at the Alfvén radius defined implicitly by $\eta\left(R_{A}\right) \equiv 1$.

For a canonical $\beta=1$ wind velocity law, explicit solution for $R_{\mathrm{A}}$ along the magnetic equator requires finding the appropriate root of

$$
\left(\frac{R_{A}}{R_{*}}\right)^{2 n}-\left(\frac{R_{A}}{R_{*}}\right)^{2 n-1}=\eta_{*},
$$

which for integer $2 n$ is just a simple polynomial, specifically a quadratic, cubic, or quartic for $n=1,1.5$, or 2 . Even for non-integer values of $2 n$, the relevant solutions can be approximated (via numerical fitting) to within a few percent by the simple general expression,

$$
\frac{R_{\mathrm{A}}}{R_{*}} \approx 1+\left(\eta_{*}+1 / 4\right)^{1 /(2 n)}-(1 / 4)^{1 /(2 n)} .
$$

For weak confinement, $\eta_{*} \ll 1$, we find $R_{\mathrm{A}} \rightarrow R_{*}$, while for strong confinement, $\eta_{*} \gg 1$, we obtain $R_{\mathrm{A}} \rightarrow \eta_{*}^{1 /(2 n)} R_{*}$. In particular, for the standard dipole case with $n=2$, we expect the strong-confinement scaling $R_{\mathrm{A}} / R_{*} \approx \eta_{*}^{1 / 4}$. 
Clearly $R_{\mathrm{A}}$ represents the radius at which the wind speed $v$ exceeds the local Alfvén speed $V_{A}$. It also characterizes the maximum radius where the magnetic field still dominates over the wind. For $\mathrm{Ap} / \mathrm{Bp}$ stars where stellar fields are of order $\mathrm{kG}, \eta_{*} \gg 1$, e.g. for $\sigma$ Ori $\mathrm{E}$ it is about $10^{7}$, implying an Alfvèn radius $\sim 60 R_{*}$. Thus, in Bp (and Ap) stars wind is trapped to large radii creating extensive magnetospheres. This also implies that X-rays from Bp stars should be intrinsically hard. But as we show in $\S 2.2$, shock retreat effects may soften it significantly.

\subsubsection{MHD Simulations}

The initial magnetohydrodynamic (MHD) simulations by ud-Doula and Owocki (2002) assumed, for simplicity, that radiative heating and cooling would keep the wind outflow nearly isothermal at roughly the stellar effective temperature. The simulations studied the dynamical competition between field and wind by evolving MHD simulations from an initial condition when a dipole magnetic field is suddenly introduced into a previously relaxed, onedimensional spherically symmetric wind.

Immediately after the introduction of the field, the dynamic interplay between the wind and the field leads to two distinct regions. Along the polar region, the wind freely streams radially outward, stretching the field lines into a radial configuration, as can be inferred from the left panel of illustrative Fig. 1. If the field is strong enough, around the magnetic equator a region of closed magnetic loops is formed wherein the flow from opposite hemispheres collides to make strong shocks, quite similar to what was predicted in the semi-analytic, fixed-field models of Babel and Montmerle (1997a). The shocked material forms a dense disk-like structure which is opaque to line-driving. But, its support against gravity by the magnetic tension along the convex field lines is inherently unstable, leading to a complex pattern of fall back along the loop lines down to the star, again as suggested by the left panel of Fig. 1 .

Note that even for weak field models with moderately small confinement, $\eta_{*} \leq 1 / 10$, the field still has a noticeable global influence on the wind, enhancing the density and decreasing the flow speed near the magnetic equator. However, shock speeds are probably not sufficiently large enough to produce any X-rays in that case.

But to model the actual X-ray emission from shocks that form from the magnetic channeling and confinement, subsequent efforts (ud-Doula, 2003; Gagné et al., 2005a) have relaxed the assumption of isothermal equation of state in earlier studies to include a detailed energy equation that follows the radiative cooling of shock-heated material. The magnetically channeled wind shock model provides excellent agreement with the diagnostics from the phase-resolved Chandra spectroscopy of $\theta^{1}$ Ori $\mathrm{C}$ (see $\S 3$ for more details).

\subsection{3. $3 D M H D$}

To get a more complete picture of the wind-field interaction, one needs ultimately full 3D MHD simulations. Us-

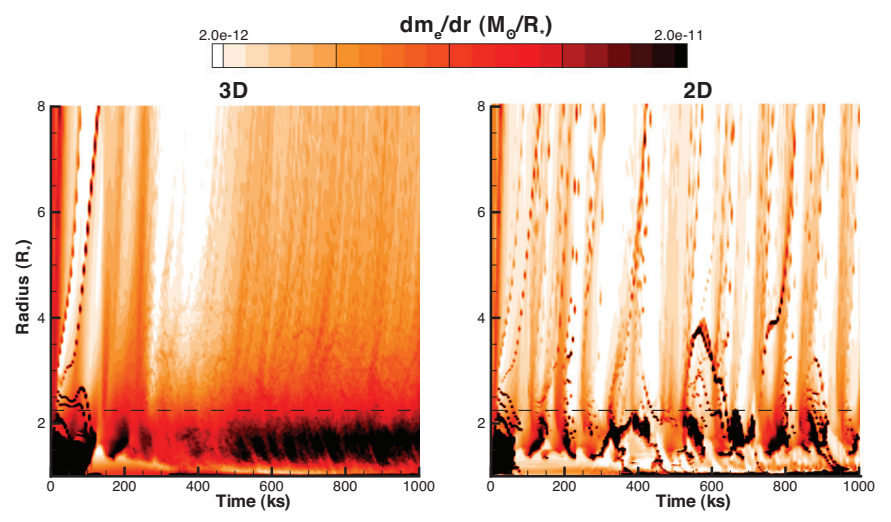

Figure 2: Equatorial mass distribution $d m_{\mathrm{e}} / d r\left(\right.$ in units $M_{\odot} / R_{*}$ ) for the azimuthally averaged 3D model (left) and for a corresponding $2 \mathrm{D}$ model (right), plotted versus radius and time, with the dashed horizontal line showing the Alfvèn radius $R_{\mathrm{A}} \approx 2.23 R_{*}$. Following a similar adjustment to the initial condition, the long-term evolution of the $2 \mathrm{D}$ models is characterized by a complex pattern of repeated strings of infall, whereas the 3D azimuthally averaged model settles into a relatively smooth asymptotic state characterized by an enhanced mass near and below the Alfvén radius. Figure reproduced from ud-Doula et al. (2013).

ing the specific parameters chosen to represent the prototypical slowly rotating magnetic $\mathrm{O} \operatorname{star} \theta^{1}$ Ori $\mathrm{C}$, for which centrifugal and other dynamical effects of rotation are negligible, ud-Doula et al. (2013) have computed the first fully 3D MHD model of its wind. The computed global structure in latitude and radius resembles that found in previous 2D simulations, with unimpeded outflow along open field lines near the magnetic poles, and a complex equatorial belt of inner wind trapping by closed loops near the stellar surface, giving way to outflow above the Alfvén radius. In contrast to this previous $2 \mathrm{D}$ work, the $3 \mathrm{D}$ simulation also shows how this complex structure fragments in azimuth, forming distinct clumps of closed loop infall within the Alfvén radius, transitioning in the outer wind to radial spokes of enhanced density with characteristic azimuthal separation of $15^{\circ}-20^{\circ}$. Applying these results in a $3 \mathrm{D}$ code for line radiative transfer, they show that emission from the associated 3D 'dynamical magnetosphere' (DM) matches well the observed $\mathrm{H} \alpha$ emission seen from $\theta^{1}$ Ori $\mathrm{C}$, fitting both its dynamic spectrum over rotational phase and the observed level of cycle-to-cycle stochastic variation. Comparison with previously developed 2D models for the Balmer emission from a dynamical magnetosphere generally confirms that time averaging over $2 \mathrm{D}$ snapshots can be a good proxy for the spatial averaging over $3 \mathrm{D}$ azimuthal wind structure, as illustrated in Fig. 2. Nevertheless, fully 3D simulations will still be needed to model the emission from magnetospheres with non-dipole field components such as $\tau$ Sco. A similar study examining X-rays predicted by this 3D MHD model is currently under way (Cohen et al., in preparation). 

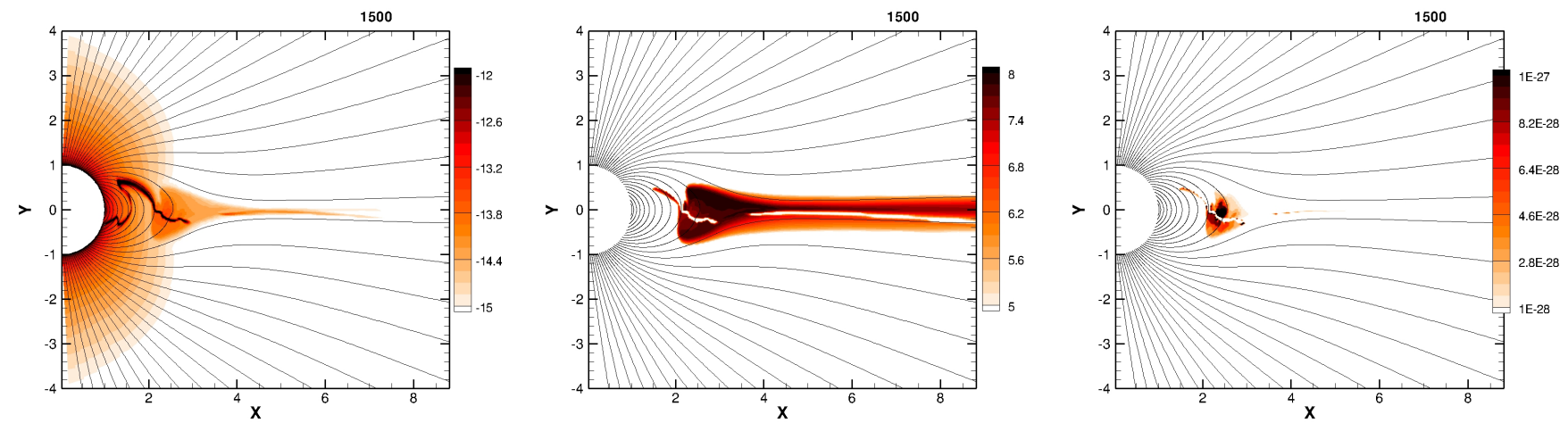

Figure 1: Colour plots of log density (left) and log temperature (middle) for arbitrary snapshot of structure in the standard model with $\eta_{*}=100$. The right panel plots the proxy X-ray emission $X E M_{T_{x}}$ (weighted by the radius $r$ ), on a linear scale for a threshold X-ray temperature $T_{x}=1.5 \mathrm{MK}$. Figure reproduced from ud-Doula et al. (2014).

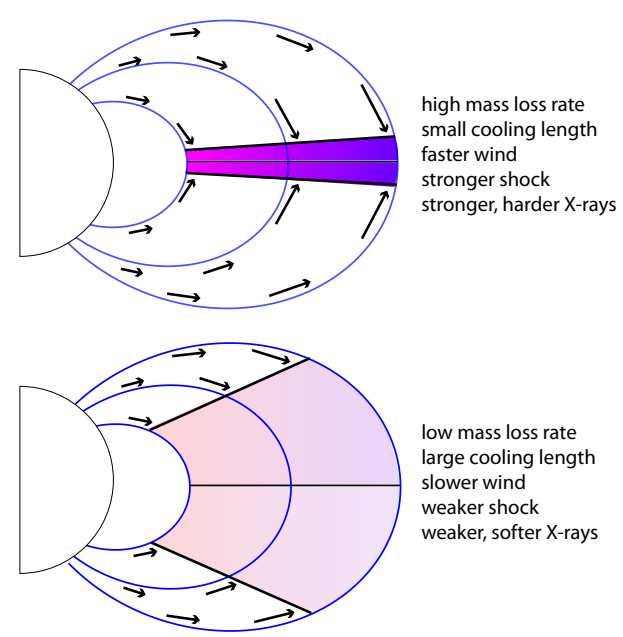

Figure 3: Schematic illustration of the "shock retreat" from inefficient cooling associated with a lower mass loss rate $\dot{M}$, showing a hemispheric, planar slice of a stellar dipole magnetic field. Wind outflow driven from opposite foot-points of closed magnetic loops is channeled into a collision near the loop top, forming magnetically confined wind shocks (MCWS). For the high $\dot{M}$ case in the upper panel, the efficient cooling keeps the shock-heated gas within a narrow cooling layer, allowing the pre-shock wind to accelerate to a high speed and so produce strong shocks with strong, relatively hard X-ray emission. For the low $\dot{M}$ case in the lower panel, the inefficient cooling forces a shock retreat down to lower radii with slower pre-shock wind, leading to weaker shocks with weaker, softer X-ray emission. Figure reproduced from ud-Doula et al. (2014).

\subsection{Shock Retreat}

Although magnetic confinement is the dominant effect in production of X-rays in magnetic massive stars, there are other secondary effects that can influence both the total luminosity and hardness of X-rays. In particular, an effect called 'shock retreat' directly related to cooling efficiency can significantly moderate X-rays in stars with low mass loss rates such as Bp stars.

As illustrated schematically in Figure 3 (ud-Doula et al., 2014, see also Figure 13 of Babel and Montmerle 1997a), the much lower mass loss rates of such B-stars also implies much less efficient cooling of the post-shock flow. When the associated cooling length, becomes comparable to the Alfvén radius, the shock location is effectively forced to "retreat" back down the loop, to a lower radius where the lower wind speed yields a weaker shock, implying then a much softer X-ray spectrum. It was shown that such shock retreat can be effectively parametrized by a cooling parameter, $\chi_{\infty} \propto V_{\infty}^{4} / \dot{M}$. A high value of this cooling parameter, $\chi_{\infty}$, implies an inefficient cooling, and, thus, a large shock retreat effect (ud-Doula et al., 2014).

In extreme low mass loss cases, value of $\chi_{\infty} \gg 1$ which may lead the shock retreat to be as large as the loop size. This can quench the wind within the closed magnetosphere suppressing the $\mathrm{X}$-ray production altogether. Here, it may be interesting to note that confined winds were also suggested to play a role in the X-ray emission of mid-B to mid-A stars, when detected (Czesla and Schmitt, 2007; Stelzer et al., 2011; Robrade and Schmitt, 2011). Indeed, after examining the ratio of X-ray to radio luminosities, Robrade and Schmitt (2011) excluded low-mass companions as the sources of X-rays. Considering a sample of Ap/Bp stars, these authors (see also Robrade 2014) further found that only stars brighter than $\sim 200 L_{\odot}$ emit $\mathrm{X}$-rays, implying the presence of a threshold in physical phenomena, whose origin could be linked to shock retreat effects.

Now, to estimate the total X-ray luminosity in typical 
OB stars, $L_{X}$, ud-Doula et al. (2014) developed a semianalytic analysis to derive a generalized "X-ray Analytic Dynamical Magnetosphere" (XADM) scaling law for Xrays emitted from such MCWS. To describe the paradigm briefly without much technical details, XADM assumes local latitudinal variation of radial mass flux at the stellar surface as prescribed by Owocki and ud-Doula (2004). The size of the magnetosphere can be readily estimated from the magnetic confinement parameter, $\eta_{*}$. It then computes shock velocity along a loop. Energy emitted as X-rays above a threshold can be estimated from kinetic energy dissipated in the shocks. Integration over the closed magnetosphere then yields the total X-ray luminosity above a threshold assuming $100 \%$ efficiency.

The resultant XADM scaling follows a very similar trend to the full magnetohydrodynamic (MHD) simulation results, but is about a factor 5 higher. Compared to the idealized steady-state emission of the analytic XADM model, the numerical MHD simulations show extensive time variability with repeated intervals of infall of cooled, trapped material, and it appears that this lowers the overall efficiency of X-ray emission to about 20 per cent of the idealized XADM prediction.

\subsection{The Effects of Rotation}

A further complication arises from rotation (not accounted for by XADM), as short-period modulations and substantial broadening in photospheric spectral lines (Conti and Ebbets, 1977; Fukuda, 1982), corresponding to projected rotation speeds of hundreds of $\mathrm{km} / \mathrm{s}$, have been detected in some massive stars.

For a non-magnetic rotating star, conservation of angular momentum in a wind outflow causes the azimuthal speed near the equator to decline outward as $v_{\phi} \sim 1 / r$, meaning that rotation effects tend to be of diminishing importance in the outer wind. However, at the stellar surface, reduced effective gravity leads to enhanced mass loss rate (ud-Doula et al., 2008, 2009) and slower wind.

By contrast, in a rotating star with a sufficiently strong magnetic field, magnetic torques on the wind can spin it up; for some region near the star, i.e., up to about the maximum loop closure radius $R_{\mathrm{c}}$ (closely related to Alfvèn radius, $R_{A}$ ), they can even maintain a nearly rigid-body rotation. As such, even for a star with surface rotation below the orbital speed, maintaining rigid rotation will eventually lead to a balance between the outward centrifugal force from rotation and the inward force of gravity.

Unsupported material at radii $r<R_{\mathrm{K}}$, Kepler radius, will tend to fall back toward the star, but any material maintained in rigid-rotation to radii $r>R_{\mathrm{K}}$ will have a centrifugal force that exceeds gravity, and so will tend to be propelled further outward, unless held down by strong enough magnetic field as is the case for Bp and some other magnetic B stars.

This complex interaction between the field and the wind naturally leads to two distinct groups of magnetic stars: "dynamical magnetospheres" (DM) and "centrifugal magnetospheres" (CM) (Sundqvist et al., 2012; Petit et al., 2013). For the former case, $R_{A}<R_{\mathrm{K}}$ and rotation is dynamically unimportant with all the supported material within the magnetosphere falling back toward the star. Notably, all Of?p stars fall within this category (their slow rotation probably comes from magnetic braking, see more details below). X-ray emission from DM is governed mainly by the magnetic confinement and shock retreat effects.

Whereas, for the latter case, $R_{A}>R_{\mathrm{K}}$ leading to corotating magnetospheres, as is the case for the rapidly rotating strong magnetic star $\sigma$ Ori E. The effect of rapid rotation on X-ray emission is currently a very much work in progress. However, preliminary studies show that the enhanced mass loss due to rotation leads to a more efficient cooling, which in turn can both increase and harden X-rays (Bard and Townsend, 2015). Here, there is a three way competition between rotation, magnetic confinement and shock retreat. Since the cooling parameter $\chi_{\infty} \propto$ $V_{\text {shock }}^{4} / \dot{M}$, the shock retreat effects might be moderated by the effects of rotation which increases $\dot{M}$ in general. But which effect ultimately dominates may depend on the details of stellar parameters of specific stars and rapidly rotating $\mathrm{B}$ stars can have a variety of X-ray luminosities. This is discussed further in $\S 3$.

Another natural consequence of interaction of magnetic field with wind and rotation is the increase in angular momentum loss from the stellar surface. It turns out that the angular momentum is carried away by not only the gas, but also by the field itself in the form of Maxwellian stress tensor. In a classical analysis, Weber and Davis (1967) showed that the total angular momentum loss can be expressed as $\dot{J}=(2 / 3) \dot{M} \Omega R_{A}^{2}$, where $\dot{M}$ is the mass loss rate, $\Omega$ is the stellar angular velocity, and $R_{A}$ is a characteristic Alfvén radius. ud-Doula et al. (2009) have shown that the typical timescale for spindown for massive magnetic $\mathrm{O}$ stars is about $1 \mathrm{Myr}$ or so. This has a direct effect on the evolution of these stars and their X-ray luminosities. Currently, all known strongly magnetic O-stars are slow rotators, with the exception of Plaskett's star where interactions within the binary are speculated to play an additional role in spinup of the primary (Grunhut et al., 2013; Palate and Rauw, 2014).

\section{Observational Perspective}

From the theoretical perspective, magnetic confinement, shock retreat and rotation effects are the main ingredients for production and explanation of X-rays in MCWS. Of course, other factors like the tilt angle of the field to the rotation axis or more generally the field geometry can also have substantial influence, as could also the presence of a companion, of stellar pulsations or of additional (azimuthal) structures in the wind. Now, let us turn our attention to what the observations actually show. We 
shall see that, despite a few exceptions, indeed these three parameters can mostly account for X-ray emissions from magnetic massive stars.

The observed scatter in X-ray luminosity of AB stars may, as in OB stars, be related to different confinement, rotation, and cooling parameter values, but better knowledge of these parameters are required before a comprehensive comparison with models can be done just like for $\mathrm{OB}$ stars, so we will not describe these objects in detail here.

\subsection{X-ray luminosity}

The X-ray luminosity of massive OB stars is well known to scale linearly with the bolometric luminosity, $\log \left[L_{\mathrm{X}} / L_{\mathrm{BO}}\right.$ -7 . However, there are some exceptions that display overluminosities, like the well-known cases of $\theta^{1}$ Ori C or $\tau$ Sco (Schulz et al., 2000; Gagné et al., 2005a; Mewe et al., 2003; Cohen et al., 2003). The discovery of magnetic fields in these objects is relatively recent, but they were found to play a key role in explaining their X-ray spectra. This overluminosity, of about one dex compared to the majority ("normal") of OB stars, was further found in many other magnetic massive stars ( $\sigma$ Ori E - Skinner et al. 2008 or the Of?p stars - Nazé et al. 2004, 2007, 2008, 2015; Petit et al. 2015). However, some other magnetic massive stars appear much fainter (Favata et al., 2009; Oskinova et al., 2011; Ignace et al., 2013; Nazé et al., 2014).

Several attempts have been made recently to understand such diversity. A first, limited survey of 11 objects by Oskinova et al. (2011) found no link between the level of X-ray luminosity and the magnetic field strength, rotational period, bolometric luminosity, or pulsational period (when existing). The general trend amongst stars (more/less luminous) seemed however qualitatively accounted for by the formula of Babel and Montmerle (1997b), $L_{\mathrm{X}} \propto$ $\dot{M} V_{\infty} B^{0.4}$.

A larger survey, covering nearly two thirds of the catalog of known magnetic OB stars of Petit et al. (2013) was undertaken by Nazé et al. (2014). The 40 targets used in the survey were scattered all over the so called "magnetic confinement-rotation diagram" (see Petit et al., 2013), limiting the biases. The situation then appeared in more detail: some of the sources (O-stars and a few B-stars) have $\log \left[L_{\mathrm{X}} / L_{\mathrm{BOL}}\right] \sim-6.2$ or equivalently $L_{\mathrm{X}} \propto \dot{M}^{0.6}$ whereas other B-stars display $L_{\mathrm{X}} \propto L_{\mathrm{Bol}}^{1.9}$ or $L_{\mathrm{X}} \propto \dot{M}^{1.4}$ (Fig. 4).

These observations are clearly at odds with the earlier Babel \& Montmerle prediction of $L_{\mathrm{X}} \propto \dot{M}$. Moreover, the level of emission predicted by this formalism is $1.8 \mathrm{dex}$ too high. However, the more recent XADM formalism, confirmed by MHD simulations, has shown much more promise (ud-Doula et al., 2014). The right panel of figure 4 shows a comparison between the observed X-ray luminosity of magnetic stars and the predicted values using the XADM model of ud-Doula et al. (2014). The dotted line illustrates the ideal model with $100 \%$ efficiency whereas the solid line indicates a scaling by $10 \%$; the grey shaded area corresponds to scalings by $5-20 \%$, a range in efficiency consistent with MHD models. The observed X-ray luminosities for most of the known magnetic stars fall within this range.

However, there are several clear exceptions (Fig. 4), five of which show observed luminosities larger than predicted. A first outlier is $\tau$ Sco (\#11) which has a complex non-dipolar field, something the current version of $\mathrm{XADM}$ does not consider as it is limited to pure dipolar cases. Three cases, $\sigma$ Ori E (\#31), HD 182180 (\#45) and HD 142184 (\#47), are rapid rotators (Petit et al., 2013). However, XADM does not take into account rapid rotation, but Bard and Townsend (2015) have shown that rotation both enhances and hardens the X-ray emission. It may be noted in this context that those three objects are also particularly hard (Nazé et al., 2014), but it should also be mentioned that the difference between rotating and non-rotating cases in Bard \& Townsend models is less than one dex, while the observed difference for those three objects is larger (about 2 dex). In addition, HD 64740, a star with similar properties (rotation rate, confinement, cooling) as $\sigma$ Ori $\mathrm{E}$ does not display a bright and hard emission, and its $L_{\mathrm{X}}$ appears in line with XADM predictions. Finally, a last exception, HD200775 (\#42) remains unexplained.

There is also one case where the observed luminosity is smaller than the predicted one. It corresponds to NGC 1624-2 (\#5), which hosts a very strong (20 kG) magnetic field. It turns out that the huge magnetosphere of this extreme magnetic star heavily attenuates its X-ray emission, making it appear less luminous and much harder than it intrinsically is (Petit et al., 2015). In fact, the estimated intrinsic luminosity is quite consistent with prediction from the XADM formalism.

\subsection{Absorption and hardness of spectrum}

Traditionally, a basic "magnetic-thus-hard" paradigm was assumed. However, one could a priori consider observed hardness of MCWS with care. Indeed, for NGC 1624-2, the apparent hardness of the X-ray spectrum is actually due to the large absorption by the dense circumstellar material $\left(N_{\mathrm{H}} \sim 1-4 \times 10^{21} \mathrm{~cm}^{-2}\right)$, the intrinsic hardness being drastically different (Petit et al., 2015). MHD simulations of such an extreme magnetosphere are presently out-of-reach, but the general properties of this object qualitatively agree with those expected from confined winds in a extreme magnetosphere as predicted by XADM paradigm (Petit et al., 2015).

However, NGC 1624-2 clearly is an extreme case. In the global survey of magnetic OB stars by Nazé et al. (2014), the amount of local absorption in spectral fits was compared to that found from general surveys of OB stars and they were found to be similar. This suggests no significantly increased absorption due to confined winds in most magnetic OB stars, which is corroborated by the monitoring of $\theta^{1}$ Ori C, where only a small increase (from 4.5 to $5 \times 10^{21} \mathrm{~cm}^{-2}$, not even formally significant considering the errors) was found (Gagné et al., 2005a). This is consistent 

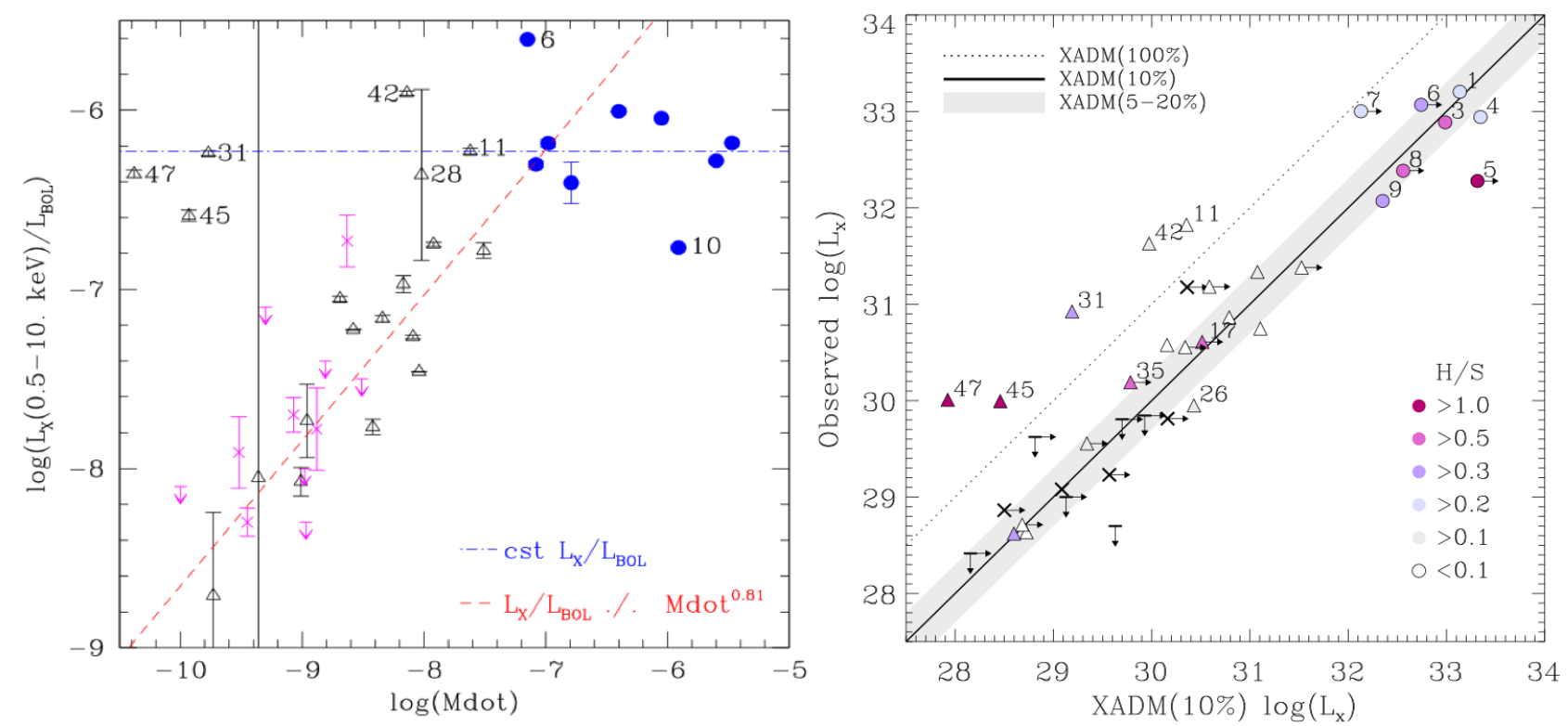

Figure 4: Observed X-ray luminosity (corrected for ISM absorption) as a function of mass-loss rate (left) and of the predicted values using the XADM model of ud-Doula et al. (2014) (right). Left: Filled blue dots correspond to O-stars, black empty triangles to B-stars, magenta crosses and downward-pointing arrows to faint detections and upper limits on the X-ray luminosity, respectively. Blue and magenta lines represent $L_{x} \propto \dot{M}^{0.6}$ and $L_{x} \propto \dot{M}^{1.4}$ relation. Right: The dotted line illustrates the ideal model with $100 \%$ efficiency whereas the solid line indicates a scaling by $10 \%$; the grey shaded area corresponds to scalings by $5-20 \%$ (a range in efficiency consistent with MHD models). The symbol shapes are the same as in the left panel, but this time symbols are colour-coded according to their measured hardness ratios. Figures reproduced from Nazé et al. (2014), numbers correspond to star IDs defined in Petit et al. (2013).

with the small density columns predicted by MHD models (see e.g. Fig. 10 of Petit et al., 2015). The observed hardness, once corrected for ISM absorption, is thus generally a good indicator of the intrinsic hardness.

Observationally, the spectrum of $\theta^{1}$ Ori C (\#3 in Fig. 4) is dominated by plasma at $\sim 2.5 \mathrm{keV}$ (Schulz et al., 2000; Gagné et al., 2005a), and hot plasma is certainly present in other stars, e.g. $\tau$ Sco (Mewe et al., 2003; Cohen et al., 2003) or Of?p stars (Nazé et al., 2004, 2007, 2008). But the hard component does not always dominate, as in Of?p stars (which usually appear soft), or may not even be detectable (e.g. $\beta$ Cep, Favata et al., 2009). Even cases of "twins" wherein a pair of stars have twin-like similar stellar parameters, revealed some surprises, for instance with one $\tau$ Sco analog being quite similar to its prototype (HD 63425) while the other one (HD 66665) was softer (Ignace et al., 2013).

Surveys by Oskinova et al. (11 stars, 2011) and Nazé et al. (40 stars, 2014) have noted such diversity of the situation. Comparison with general OB stars surveys further reveals that magnetic O-stars appear at least somewhat harder than "normal" O-stars while magnetic B-stars seemed rather softer than usual B-stars (Nazé et al., 2014). The latter conclusion may however be biased by the different population sampled, the observed magnetic B-stars sampling lower X-ray luminosities than in surveys. Nevertheless, no correlation could be found between hardness and magnetic or stellar parameters (Nazé et al., 2014). In particular, the prediction of harder X-ray emission in case of stronger magnetic confinement, larger mass-loss rate, or higher rotation rate was not detected.

\subsection{X-ray Variability}

By design, theoretical predictions such as XADM are time independent, although fully self-consistent MHD models do suggest certain level of time variability in X-rays and other bands. Observationally, several types of variabilities associated with magnetically confined winds have been detected. In general, these variabilities do not change global properties of X-ray emission discussed above, but they do show how complex the interplay between the field and wind can be.

\subsubsection{Flares}

The first type of possible variations is short-term X-ray flaring. MHD models of dynamical magnetospheres show a complex temporal behaviour, with stochastic episodes of heating and infall back on the star - but they are limited to small regions, hence are smoothed out in 3D magnetospheres (ud-Doula et al., 2013). In centrifugal magnetospheres, however, the trapped material accumulates and, to keep the balance, some ejection needs to take place, through "centrifugal" breakouts involving magnetic reconnection on the outer edges of magnetospheres (Townsend and Owocki, 2005; ud-Doula et al., 2006). This should generate flares and such events have been recorded in $\sigma$ Ori E by ROSAT, XMM, and Chandra (Groote and Schmitt, 
2004; Sanz-Forcada et al., 2004; Skinner et al., 2008; Caballero et al., 2009), but their interpretation was much debated: is it intrinsic to $\sigma$ Ori $\mathrm{E}$ or due to an unseen lowmass companion? Several arguments can be put forward for the former scenario. For example, the decay part of the flare lightcurve suggests a size of $\sim 1 R_{*}$ for the region involved in the event (considering $R_{*}$ to be the radius of $\sigma$ OriE), which is reasonable in the context of MCWS (Groote and Schmitt, 2004; Mullan, 2009) but this size would correspond to $10-20 R_{*}$ when considering the radius of a K-type companion, too large for a flare associated to an active coronal region in the companion (Mullan, 2009). In addition, while flares are typical for coronal sources and their luminosity is compatible with that observed, flares of the same magnitude as those observed occurred rarely for low-mass stars and their typical recurrence timescale would have prevented several observation of such events over two decades (Mullan, 2009). On the other hand, Bouy et al. (2009) did detect the presence of a low-mass companion and the analysis of the source position during the Chandra-HRC flaring episode favors the low-mass companion as origin of the flare (Petit et al., 2012). Furthermore, the magnetospheric mass derived by Townsend et al. (2013) appears to be two orders of magnitude below the level required for centrifugal breakouts to happen as initially suggested by ud-Doula et al. (2006). A rather similar situation was found for the magnetic Ap star IQ Aur, where the estimated size of the flaring region, the position of the X-ray source, the soft character of the recorded Xrays (compared to young active stars), and the high $\mathrm{X}$-ray to radio luminosity ratio favor an origin of the flare in the Ap star, but the influence of a low-mass companion cannot be formally excluded (Robrade and Schmitt, 2011). Finally, the detection of several flares in HD 47777 was also reported by Nazé et al. (2014) but again, it remains to be confirmed that they are actually associated with the magnetically confined winds of the star, rather than with an yet unknown low-mass companion of the star.

\subsubsection{Rotational modulation}

A second type of variation is rotational modulation, expected to occur on longer timescales than flaring. This type of variability strongly depends on the magnetospheric geometry and the orientation of the star with respect to the line of sight. Observations have been analyzed to reveal such changes.

Amongst magnetic OB stars, $\tau$ Sco displays a rather complex field geometry, with one model restricted regions of densely concentrated closed loops asymmetrically distributed on the stellar surface (Donati et al., 2006). Strong phase-locked variability could thus be expected (40\% modulation, Donati et al., 2006), as some regions appear in plain view at some phases and are occulted at others, but a dedicated Suzaku monitoring found at best very low amplitude changes in flux (a few percents and mostly in soft band, Ignace et al., 2010). This may be explained if the distribution of hot gas is different (e.g. in smaller loops) from the magnetospheric geometry derived from optical spectropolarimetry. Certainly more detailed investigation is needed before this observational result can fully be understood.

With a simpler, mostly dipolar magnetic field, other magnetic OB stars should show changes related to the viewing angle on the confined winds (i.e. alternative edgeon/face-on view). Indeed, X-ray variations simultaneous with optical changes have been detected in at least four well-documented cases: $\theta^{1}$ Ori C (Gagné et al., 2005a; Stelzer et al., 2005, and references therein), HD 191612 (Nazé et al., 2010), CPD - 28 2561 (Nazé et al., 2015), and NGC1624-2 (Petit et al., 2015). For these objects, the X-ray emission and the $\mathrm{H} \alpha$ emission appear brighter when the confined winds are seen face-on.

In the $\mathbf{X}$-ray domain, variation amplitudes $\left(F_{\max } / F_{\min }-\right.$ 1) typically reach $40-100 \%$ for $\theta^{1}$ Ori C , HD 191612, NGC16242 , and CPD $-28^{\circ} 2561$ as well as NU Ori (Nazé et al., 2014) and Tr16-22 (Nazé et al., 2014), but the amplitude appears smaller in a few other cases. For example, outside flares, the X-ray lightcurve of $\sigma$ Ori E shows limited variability: Skinner et al. (2008, see in particular their Fig. 5) detected variations with an amplitude $\left(F_{\max } / F_{\min }-1\right) \sim$ $30 \%$ but at the $1.4 \%$ significance level only - the amplitude is in fact comparable to the noise and requires confirmation. Similarly, the X-ray flux of $\beta$ Cep changes by at most $10 \%$ and its phase-locked nature still need to be established (Favata et al., 2009; Nazé et al., 2014). Finally, a stable X-ray emission is observed for HD 148937, which is not surprising: it is always seen nearly pole-on, hence the view on the confined winds of this oblique rotator does not vary much, explaining the low amplitude changes in the optical domain and the absence of detectable variations in X-rays (Nazé et al., 2014).

The phase-locked variability observed in X-rays could be explained in two different ways. On the one hand, it could be related to absorption. For example, if Xrays arise in the dense equatorial regions, then some absorption of that emission could take place, reducing the observed (soft) X-ray flux especially when these regions are seen edge-on. However, to produce a large flux decrease, this absorption increase would become noticeable in spectral fitting. This is the case of the extremely magnetic O-star NGC1624-2, where the very dense magnetosphere produces a strong and variable additional absorption $\left(1-4 \times 10^{22} \mathrm{~cm}^{-2}\right.$, Petit et al. 2015). The spectrum with minimum emission clearly lacks soft X-ray photons when compared to that of the maximum emission phase, and occultation effects (see below) could only be responsible at most for a $20 \%$ variation in (intrinsic) flux. However, this situation is exceptional, as the local, additional absorption due to confined winds generally remains low, as mentioned before, and no significant increase in absorption for the edge-on configuration was reported in other stars (Gagné et al., 2005a; Nazé et al., 2014).

On the other hand, flux variations in magnetic stars could be related to regular occultation of the emission re- 
gions by the stellar body. This is the favored explanation and it could explain the scatter in amplitude change. For example, the small variations of $\beta$ Cep are compatible with occultation of hot plasma located at $4-6 R_{*}$, the position derived from high-resolution spectra (Favata et al., 2009). $\sigma$ Ori $\mathrm{E}$ has a very large magnetosphere (about $31 \mathrm{R}_{*}$ for the Alfvén radius), which minimizes the impact of occultations and could contribute to explain the lack of clear variation detection (Nazé et al., 2014). However, as the flux changes are often accompanied by changes in spectral shape, this would imply that occultation affects plasma of different temperatures in different ways as could be expected, e.g., if thermal stratification exists (Nazé et al., 2014). In this context, it may be worth noting the variety of behaviours, which remains to be explained: HD 191612, Tr16-22, CPD - 28 2561 , and NU Ori appear harder when brighter, while $\theta^{1}$ Ori $\mathrm{C}$ appears to be softer while brighter (Nazé et al., 2014).

To assess the occultation explanation, we can model the confined winds by a simple optically-thin structure near the star, and predict the occultation degree for various situations. We tested several geometries: a disk up to Alfvén radius $R_{A}$ and a ring from $R_{A}-R_{*}$ to $R_{A}$, which better reproduce the geometry of emitting regions observed in MHD simulations. Figure 5 shows how occultation depends on geometry (a disk is more occulted than a ring) and its variation with phase depends on orientation (i.e. for various inclination and obliquity combinations). Most important is the location of the plasma, of course. Considering a ring geometry in agreement with MHD models yields a relative flux variation $F_{\min } / F_{\max }$ of $\sim 0.83$, or $\left[F_{\max } / F_{\min }\right]-1 \sim 20 \%$, for the case of $\theta^{1}$ Ori $\mathrm{C}$ while the observed value is $\left[F_{\max } / F_{\min }\right]-1 \sim 50 \%$. Similar conclusions are reached for CPD $-28^{\circ} 2561$ or HD 191912. To get higher occultation degrees, one could change the plasma location, as structures closer to the star are more occulted than those further away. To get the observed value of $\theta^{1}$ Ori C, one would however need to consider a ring of negligible radius located at $1.15 R_{*}$, less than half the Alfvén radius but also much less than the plasma location derived by He-like triplets (Gagné et al., 2005b), which seems dubious. For cases like Tr16-22, where $\left[F_{\max } / F_{\min }\right]-1 \sim 100 \%$, then the hot plasma would need to be at the photosphere. Clearly, the simple model is underevaluating occultation, most probably because of its simplicity. Considering some limited absorption may help, but a fully optically-thick case would probably be inappropriate because of its phase shift ${ }^{2}$.

\footnotetext{
${ }^{2}$ Donati et al. (2001) have envisaged the case of confined winds being fully optically thick. In that case, the X-ray flux would be maximum when the confined winds are seen edge-on (i.e. when the optical emission are minimum) as the emission from both hemispheres would then be seen, while only the emission of "upper half" of the confined winds would be observable at other phases, that of the "lower half" being fully absorbed. While flux variations by a factor of two have been detected, it must be noted that a shift by half a cycle between $\mathrm{X}$-ray and optical maxima has not been reported, up to now.
}
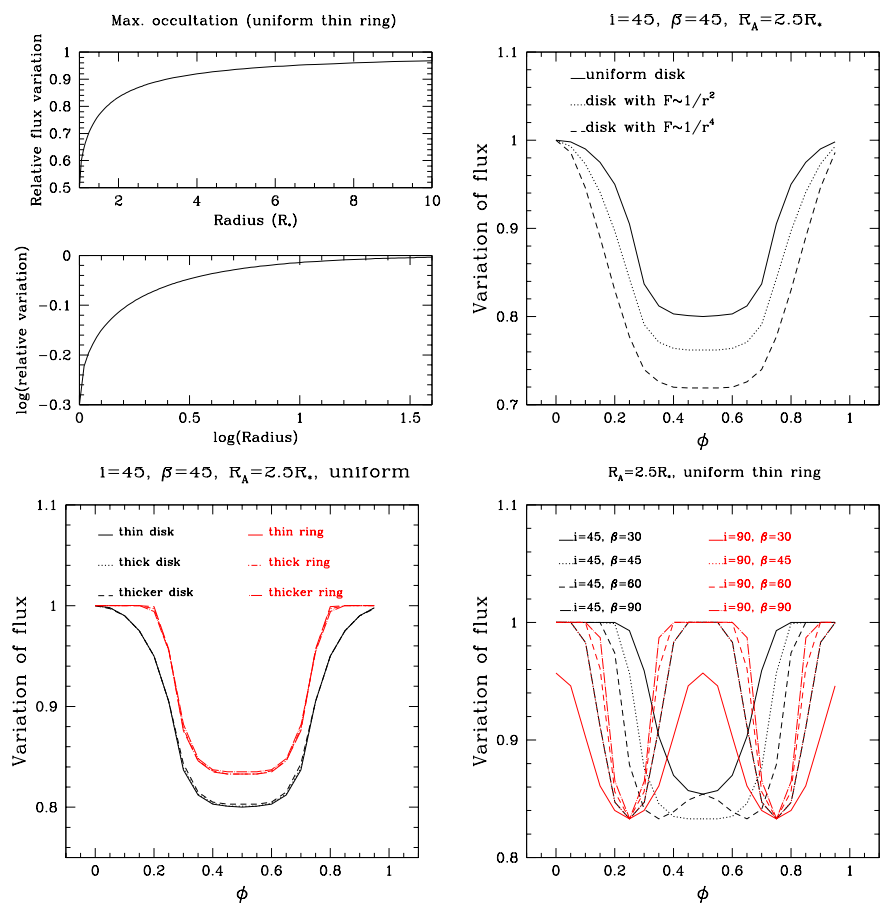

Figure 5: Top left: Evolution with radius of the minimum value of the apparent flux, relative to the unocculted case, of a ring of negligible $\Delta r$ lying in the magnetic equatorial plane. Note that, depending on orientation, structures very close to the star may remain always partially occulted throughout the cycle, hence the y-axis value does not necessarily represent the observed $F_{\min } / F_{\max }$, it is only the minimum possible. Top right: Variation of relative flux with phase, for different brightness functions (uniform brightness, evolution as $r^{-2}$ or $\left.r^{-4}\right)$. The Alfvén radius, inclination, and obliquity are typical of $\theta^{1}$ Ori C. Bottom left: Variation of relative flux with phase, for different geometries. "Disk" is a structure extending from $R_{*}$ to $R_{A}$, "ring" an annuli extending from $R_{A}-R_{*}$ to $R_{A}$; "Thin" corresponds to structures lying in the magnetic equator only, "thick" applies to structures with a total thickness of $0.3 R_{*}$, "thicker" for thicknesses of $0.5 R_{*}$. The Alfvén radius, inclination, and obliquity are typical of $\theta^{1}$ Ori C. Bottom right: Variation of relative flux with phase, for different combinations of inclination and obliquity but a similar geometry (thin ring, with $R_{A}=2.5 R_{*}$ ). This agrees well with observations, e.g. the single minimum of $\theta^{1}$ Ori C vs. the two minima of $\mathrm{CPD}-28^{\circ} 2561$. 
3.4. Structure of confined winds, as revealed by high-resolution soft X-rays in these objects (see above), but it cannot be spectra

High-resolution spectra can yield a wealth of information. With current instrumentation, line widths and shifts can be evaluated with precisions down to a few tens $\mathrm{km} \mathrm{s}^{-1}$ in the most favorable cases (a few hundreds $\mathrm{km} \mathrm{s}^{-1}$ more typically). Furthermore, the comparison of lines from Hlike and He-like ions and of components of fir triplets of He-like ions constrain the temperature and location of the emitting plasma. However, such measurements are currently only possible for the brightest X-ray sources, so that few magnetic massive stars have been investigated in this respect ( $\tau$ Sco - Mewe et al. 2003; Cohen et al. 2003, $\theta^{1}$ Ori C - Schulz et al. 2000; Gagné et al. 2005a,b, HD 191612 - Nazé et al. 2007, HD 148937 - Nazé et al. 2008, 2012, $\beta$ Cep - Favata et al. 2009, IQ Aur - Robrade and Schmitt 2011).

Within noise limitations, the X-ray lines of magnetic massive stars were found to be symmetric, and globally unshifted. This agrees well with MHD models. In the case of $\theta^{1}$ Ori C, global fitting however suggests small variations in velocity (Gagné et al., 2005a): from $-75 \mathrm{~km} \mathrm{~s}^{-1}$ when the star is seen pole-on to about $100 \mathrm{~km} \mathrm{~s}^{-1}$ when seen edge-on. This change needs to be confirmed as the errors are large but also because one cannot exclude a stochastic variation when only a single observation per phase is available. If further observations provide evidence that velocity varies with phase, then refinement of models will be needed, as no such changes are currently predicted (Gagné et al., 2005a).

Reported widths of X-ray lines largely depend on object and ion considered. The narrowest widths, so far, were found for $\beta$ Cep, whose lines are dominated by instrumental resolution, yielding only upper limit on intrinsic widths $\left(<600 \mathrm{~km} \mathrm{~s}^{-1}\right.$, Favata et al., 2009). Larger widths, $F W H M \sim 600-800 \mathrm{~km} \mathrm{~s}^{-1}$, were reported for ions with high ionization potential $(\mathrm{Mg}, \mathrm{Si}, \mathrm{S})$ in $\tau \mathrm{Sco}, \theta^{1}$ Ori $\mathrm{C}$, and HD 148937, three stars with more rapid winds than $\beta$ Cep. Such widths are much smaller than observed for "normal" O-type stars $\left(F W H M \sim v_{\infty}\right)$, where lines arise in embedded wind shocks distributed all over the wind, hence cover a larger velocity range. They indicate formation in slowlymoving plasma, in agreement with the confined winds scenario. However, most MHD models predict even narrower lines (Gagné et al., 2005a).

Furthermore, lines from ions with lower ionization potential, notably oxygen, appear broader $(F W H M \sim 1800$ $2000 \mathrm{~km} \mathrm{~s}^{-1}$ Gagné et al., 2005a; Nazé et al., 2007, 2008). These lines are associated with cooler plasma, which could have a different origin than hotter plasma. For example, the dominant hot plasma in $\theta^{1}$ Ori $\mathrm{C}$ is thought to arise in confined winds while the cooler one could arise in embedded wind shocks as in normal O-stars (Gagné et al., 2005a). This dual origin could be supported by the different temperatures derived from the different ions (Schulz et al., 2000). In Of?p stars, however, the spectra are dominated by the cooler component, i.e. confined winds emit excluded that current errors, which are large, are somewhat blurring the picture.

In He-like triplets, the forbidden line is suppressed when the density is high or the UV radiation is intense. In the case of massive stars, the latter effect is the most important one and, thanks to dilution with distance, enables us to locate the emitting region. In $\tau$ Sco, $\theta^{1}$ Ori C, HD 148937 , and $\beta$ Cep, the start of the emitting region is found to be close to the photosphere, at radii $r \sim 1.5-3 R_{*}$ for the first three stars and $r \sim 4-6 R_{*}$ for the latter case. These values are only slightly lower than the corresponding Alfvén radii of these stars, they thus appear qualitatively compatible with MHD simulations. In IQ Aur, the forbidden line was found to be normal, suggesting a formation radius larger than $7 R_{*}$ (Robrade and Schmitt, 2011) - despite a large value, this also appears compatible with the supposed location of confined winds in this star.

\section{Discussion, Conclusion and Future Outlook}

The discovery of magnetism in massive $\mathrm{O}$ stars is a relatively recent phenomenon although magnetism in $\mathrm{B}$ stars was known for much longer. This led to a number of new observational campaigns such as MiMeS (Wade et al., 2012) or BOB (Morel et al., 2015) that increased the total number of known magnetic OB stars significantly. There have also been some theoretical developments in an attempt to understand this increasingly larger group of stars. In this brief review, we discussed specifically the X-ray emission from these objects.

Although there are some outliers, semi-analytic scaling of XADM paradigm can correctly estimate X-ray luminosities from most magnetic OB stars. A phenomenon called shock retreat can reduce the X-ray luminosity, and for the case of B stars with extremely low mass-loss rate, it may even quench the wind within the closed magnetosphere. Rotation can counter some of the effects, but much work remains to be done in order to fully understand these complex objects.

As the number of magnetic detections increases with time, future X-ray missions will certainly enlarge the samples. Indeed, most of the results presented here come from a relatively small size sample while a large number of parameters are involved in the confined wind phenomenon and its observations (magnetic strength, magnetic geometry, inclination of the system, and stellar parameters such as temperature, rotation rate, wind velocity and mass-loss rate). Notably, only about 10 magnetic O-stars are currently known and have been studied in X-rays. Moreover, the sensitivity of current facilities does not allow a large study of magnetic massive stars at high spectral resolution, nor the detection of very faint X-ray emission (e.g. $\mathrm{Ap} / \mathrm{Bp}$ stars). This certainly poses a challenge to understand these object fully and with confidence.

The advent of the new X-ray mission Athena+ (Nandra et al., 2013) may notably enable us to study con- 
fined winds in radically different environments like lowmetallicity galaxies such as the Magellanic Clouds, where massive stars are known to display weaker stellar winds. Such new data would allow to test our current models on vastly different cases, thus improving their reliability. The very first extragalactic magnetic massive candidates have already been proposed (Maitzen et al., 2001; Paunzen et al., 2011; Nazé et al., 2015; Walborn et al., 2015), so that several targets will become available in the next decade for such studies.

Another important observational information comes from monitoring the X-ray emission. It is a natural way to probe the magnetosphere, and it provides strong constraints on its hot plasma content. Unfortunately, such programs are difficult to obtain because of their length and the need of time-constraints, therefore only few objects could be followed in detail. Moreover, when a monitoring exists, it generally covers a single cycle or less - it is thus currently difficult to separate potential stochastic changes from periodic ones. With a better sensitivity, such as foreseen for Athena+, shorter exposures will be needed, reducing the total duration hence facilitating the gathering of multiple exposures.

Finally, high spectral resolution data also provide crucial information by allowing detailed studies of the X-rays associated to confined winds. Currently, only a few such spectra were obtained, and they did not yet allow analyses of the line profiles, as lines are barely resolved. Future facilities such as the European Space Agency's Athena+ observatory (Nandra et al., 2013) should provide access not only to high spectral resolution for more objects but also to better spectral resolution than currently available. This will allow us to pinpoint the properties of a larger sample with better quality, further constraining the hot plasma distribution and kinematics. Of utmost interest in this context are monitoring performed at high resolution. The behaviour of different lines will then be compared, e.g. those from ions with low-ionization potential and those associated with high ionization potential. Any difference between them will indicate different emission processes, which will be identified thanks to detailed line profile studies. Furthermore, if velocity shifts such as tentatively identified in $\theta^{1}$ Ori $\mathrm{C}$ are confirmed, then detailed tomographic analyses will become possible, enabling the reconstruction of the magnetospheric activity kinematics.

Along with the observational developments, theory must also keep up the pace. Currently, most of the fully consistent MHD simulations are limited to $2 \mathrm{D}$. One exception is the 3D MHD study of $\mathrm{H} \alpha$ emission from $\theta^{1}$ Ori $\mathrm{C}$ by udDoula et al. (2013). Since rotation in this case was dynamically unimportant, rotation and field axes were assumed to be aligned. However, in many other cases rotation is dynamically important and the field is tilted with respect to the rotation axis. Such oblique rotator cases must be modelled using full 3D MHD, something currently missing. Moreover, for stars for which magnetic confinement parameter $\eta_{*} \gg 1$, MHD cannot be used due to high Alfvèn speed that forces Curant time to be impractically small, rendering such numerical simulations essentially impossible.

In such extreme cases, alternate methods must be employed. Townsend and Owocki (2005) present a semianalytic method for modelling the circumstellar environment of early-type stars, which is essentially a generalized extension of Babel and Montmerle model. They assume that the magnetic field lines remain rigid and corotate with the star without being influenced by the dynamics of the wind. This is essentially equivalent to the assumption of $\eta_{*} \rightarrow \infty$. This Rigidly Rotating Magnetosphere (RRM) model can be applied to an arbitrary field geometry and tilt angle between the field and rotation axes. However, there are no dynamic forces involved in this model, instead effective gravitational + centrifugal potential is calculated based on the constrained motion of the plasma. Accumulation surfaces are assumed to lie along the location of minimum effective potential.

As a further improvement to the RRM model, Townsend et al. (2007) introduced a new Rigid-Field Hydrodynamics (RFHD) method to modelling the circumstellar environments of strongly magnetic massive stars as defined above. Just like in the RRM method, the field lines are treated as rigid, but now the flow along the lines is computed self-consistently using hydrodynamical equations including line force due to radiation. In this ansatz, flow along each each field line is considered to be an independent 1D flow. They perform a large number of such 1D calculations for differing field lines, then piece them together to build up a time-dependent 3D model of a magnetosphere.

This method is now superseded by Arbitrary RigidField Hydrodynamics (ARFHD) method which now allows any configuration of self-consistent magnetic topology. It also improves some of the numerical algorithms and radiative cooling and includes thermal conduction (Bard and Townsend, 2015). Since the flow along each field line can be solved independently of other field lines, the computational cost of this approach is a fraction of an equivalent full MHD simulation. However, this method suppresses any coupling between the flow on adjacent field lines which in practice may have some important ramifications. As such, a new, improved method will be needed in the future.

\section{Acknowledgements}

AuD acknowledges support by NASA through Chandra Award number TM4-15001A and 16200111 issued by the Chandra X-ray Observatory Center which is operated by the Smithsonian Astrophysical Observatory for and behalf of NASA under contract NAS8- 03060. AuD also acknowledges support for Program number HST-GO-13629.008-A provided by NASA through a grant from the Space Telescope Science Institute, which is operated by the Association of Universities for Research in Astronomy, Incorporated, under NASA contract NAS5-26555. YN acknowl- 
edge support from the Fonds National de la Recherche Scientifique (Belgium), the PRODEX XMM contract, and an ARC grant for Concerted Research Action financed by the Federation Wallonia-Brussels.

\section{References}

J. Babel, T. Montmerle, On the periodic X-ray emission from the O7 V star $\theta^{1}$ Orionis C, ApJ 485 (1997a) 29.

J. Babel, T. Montmerle, X-ray emission from Ap-Bp stars: A magnetically confined wind-shock model for IQ Aur., A\&A 323 (1997b) 121-138.

C. Bard, R. Townsend, X-rays From Centrifugal Magnetospheres in Massive Stars, in: IAU Symposium, volume 307 of IAU Symposium, 2015, pp. 449-450. doi:10.1017/S1743921314007352.

T. W. Berghoefer, J. H. M. M. Schmitt, R. Danner, J. P. Cassinelli, $\mathrm{X}$-ray properties of bright OB-type stars detected in the ROSAT all-sky survey., A\&A 322 (1997) 167-174.

H. Bouy, N. Huélamo, E. L. Martín, F. Marchis, D. Barrado Y Navascués, J. Kolb, E. Marchetti, M. G. Petr-Gotzens, M. Sterzik, V. D. Ivanov, R. Köhler, D. Nürnberger, A deep look into the cores of young clusters. I. $\sigma$-Orionis, A\&A 493 (2009) 931-946. doi:10.1051/0004-6361:200810267. arXiv:0808.3890.

J. A. Caballero, J. López-Santiago, E. de Castro, M. Cornide, X-Ray Variability of $\sigma$ Orionis Young Stars as Observed with ROSAT, AJ 137 (2009) 5012-5021. doi:10.1088/0004-6256/137/6/5012. arXiv:0903.4814.

T. Chlebowski, X-ray emission from O-type stars - Parameters which affect it, ApJ 342 (1989) 1091-1107. doi:10.1086/167667.

D. H. Cohen, G. E. de Messières, J. J. MacFarlane, N. A. Miller, J. P. Cassinelli, S. P. Owocki, D. A. Liedahl, High-Resolution Chandra Spectroscopy of $\tau$ Scorpii: A Narrow-Line X-Ray Spectrum from a Hot Star, ApJ 586 (2003) 495-505. doi:10.1086/367553. arXiv:astro-ph/0211412.

P. S. Conti, D. Ebbets, Spectroscopic studies of O-type stars. VII. Rotational velocities $\mathrm{V}$ sin i and evidence for macroturbulent motions., ApJ 213 (1977) 438-447.

S. Czesla, J. H. H. M. Schmitt, Are magnetic hot stars intrinsic Xray sources?, A\&A 465 (2007) 493-499. doi:10.1051/0004-6361: 20065890. arXiv:astro-ph/0703524.

J.-F. Donati, I. D. Howarth, M. M. Jardine, P. Petit, C. Catala, J. D. Landstreet, J.-C. Bouret, E. Alecian, J. R. Barnes, T. Forveille, F. Paletou, N. Manset, The surprising magnetic topology of $\tau$ Sco: fossil remnant or dynamo output?, MNRAS 370 (2006) 629-644. doi:10.1111/j.1365-2966.2006.10558. x. arXiv:arXiv:astro-ph/0606156.

J.-F. Donati, G. A. Wade, J. Babel, H. f. Henrichs, J. A. de Jong, T. J. Harries, The magnetic field and wind confinement of $\beta$ Cephei: New clues for interpreting the Be phenomenon?, MNRAS 326 (2001) 1265-1278.

F. Favata, C. Neiner, P. Testa, G. Hussain, J. Sanz-Forcada, Testing magnetically confined wind shock models for $\beta$ Cephei using XMM-Newton and Chandra phase-resolved X-ray observations, A\&A 495 (2009) 217-229. doi:10.1051/0004-6361: 20078529. arXiv:0806.2275.

I. Fukuda, A statistical study of rotational velocities of the stars, PASP 94 (1982) 271-284.

M. Gagné, M. E. Oksala, D. H. Cohen, S. K. Tonnesen, A. ud-Doula, S. P. Owocki, R. H. D. Townsend, J. J. MacFarlane, Chandra HETGS Multiphase Spectroscopy of the Young Magnetic O Star $\theta^{1}$ Orionis C, ApJ 628 (2005a) 986-1005. doi:10.1086/430873.

M. Gagné, M. E. Oksala, D. H. Cohen, S. K. Tonnesen, A. ud-Doula, S. P. Owocki, R. H. D. Townsend, J. J. MacFarlane, Erratum: Chandra HETGS Multiphase Spectroscopy of the Young Magnetic O Star $\theta^{1}$ Orionis C, ApJ 634 (2005b) 712-713. doi:10.1086/ 496876.

D. Groote, J. H. M. M. Schmitt, Discovery of X-ray flaring on the magnetic Bp-star $\sigma$ Ori E, A\&A 418 (2004) 235-242.

J. H. Grunhut, G. A. Wade, M. Leutenegger, V. Petit, G. Rauw, C. Neiner, F. Martins, D. H. Cohen, M. Gagné, R. Ignace,
S. Mathis, S. E. de Mink, A. F. J. Moffat, S. Owocki, M. Shultz, J. Sundqvist, MiMeS Collaboration, Discovery of a magnetic field in the rapidly rotating O-type secondary of the colliding-wind binary HD 47129 (Plaskett's star), MNRAS 428 (2013) 1686-1695. doi:10.1093/mnras/sts153. arXiv: 1209.6326.

R. Ignace, L. M. Oskinova, M. Jardine, J. P. Cassinelli, D. H. Cohen, J.-F. Donati, R. H. D. Townsend, A. ud-Doula, A Multiphase Suzaku Study of X-rays from $\tau$ Sco, ApJ 721 (2010) 1412-1420. doi:10.1088/0004-637X/721/2/1412. arXiv: 1008.1552.

R. Ignace, L. M. Oskinova, D. Massa, A report on the X-ray properties of the $\tau$ Sco-like stars, MNRAS 429 (2013) 516-522. doi:10.1093/mnras/sts358. arXiv: 1211.0861.

H. M. Maitzen, E. Paunzen, O. I. Pintado, On the detection of the first extragalactic classical chemically peculiar stars, A\&A 371 (2001) L5-L8. doi:10 .1051/0004-6361:20010431.

R. Mewe, A. J. J. Raassen, J. P. Cassinelli, K. A. van der Hucht, N. A. Miller, M. Güdel, High-resolution X-ray spectroscopy of tau Scorpii (B0.2V) with XMM-Newton, A\&A 398 (2003) 203-211. doi:10.1051/0004-6361:20021577.

T. Morel, N. Castro, L. Fossati, S. Hubrig, N. Langer, N. Przybilla, M. Schöller, T. Carroll, I. Ilyin, A. Irrgang, L. Oskinova, F. R. N. Schneider, S. S. Díaz, M. Briquet, J. F. González, N. Kharchenko, M.-F. Nieva, R.-D. Scholz, A. de Koter, W.-R. Hamann, A. Herrero, J. Maíz Apellániz, H. Sana, R. Arlt, R. Barbá, P. Dufton, A. Kholtygin, G. Mathys, A. Piskunov, A. Reisenegger, H. Spruit, S.-C. Yoon, The B Fields in OB Stars (BOB) Survey, in: IAU Symposium, volume 307 of IAU Symposium, 2015, pp. 342-347. doi:10.1017/S1743921314007054. arXiv:1408.2100.

D. J. Mullan, Flares on a Bp Star, ApJ 702 (2009) 759-766. doi:10. 1088/0004-637X/702/1/759.

K. Nandra, D. Barret, X. Barcons, A. Fabian, J.-W. den Herder, L. Piro, M. Watson, C. Adami, J. Aird, J. M. Afonso, et al., The Hot and Energetic Universe: A White Paper presenting the science theme motivating the Athena+ mission, ArXiv e-prints (2013). arXiv: 1306.2307.

Y. Nazé, P. S. Broos, L. Oskinova, L. K. Townsley, D. Cohen, M. F. Corcoran, N. R. Evans, M. Gagné, A. F. J. Moffat, J. M. Pittard, G. Rauw, A. ud-Doula, N. R. Walborn, Global X-ray Properties of the O and B Stars in Carina, ApJS 194 (2011) 7. doi:10.1088/ 0067-0049/194/1/7. arXiv:1103.0101.

Y. Nazé, V. Petit, M. Rinbrand, D. Cohen, S. Owocki, A. udDoula, G. A. Wade, X-Ray Emission from Magnetic Massive Stars, ApJS 215 (2014) 10. doi:10.1088/0067-0049/215/1/10. arXiv: 1409.1690.

Y. Nazé, G. Rauw, A. M. T. Pollock, N. R. Walborn, I. D. Howarth, Towards an understanding of the Of?p star HD191612: phase-resolved multiwavelength observations, MNRAS 375 (2007) 145-153. doi:10.1111/j.1365-2966.2006.11270. x. arXiv:astro-ph/0611230.

Y. Nazé, G. Rauw, J.-M. Vreux, M. De Becker, HD 108: The mystery deepens with XMM-Newton observations, A\&A 417 (2004) 667677. doi:10.1051/0004-6361:20034422. arXiv : astro-ph/0402480.

Y. Nazé, J. O. Sundqvist, A. W. Fullerton, A. ud-Doula, G. A. Wade, G. Rauw, N. R. Walborn, UV and X-ray monitoring of CPD -28 2561, ArXiv e-prints (2015). arXiv:1506.08572.

Y. Nazé, A. Ud-Doula, M. Spano, G. Rauw, M. De Becker, N. R. Walborn, New findings on the prototypical Of?p stars, A\&A 520 (2010) A59. doi:10.1051/0004-6361/201014333. arXiv:1006.2054.

Y. Nazé, G. A. Wade, V. Petit, Modulated X-ray emission of the magnetic O8.5V-star Tr16-22, A\&A 569 (2014) A70. doi:10.1051/ 0004-6361/201424416. arXiv: 1408.6098.

Y. Nazé, N. R. Walborn, N. Morrell, G. A. Wade, M. K. Szymański, Photometric identification of the periods of the first candidate extragalactic magnetic massive stars, A\&A (2015). arXiv: 1503.07654.

Y. Nazé, N. R. Walborn, G. Rauw, F. Martins, A. M. T. Pollock, H. E. Bond, HD 148937: A Multiwavelength Study of the Third Galactic Member of the Of?p Class, AJ 135 (2008) 1946-1957. doi:10.1088/0004-6256/135/5/1946. arXiv:0803.0605.

Y. Nazé, S. A. Zhekov, N. R. Walborn, High-resolution X-Ray Spec- 
troscopy of the Magnetic Of?p Star HD 148937, ApJ 746 (2012) 142. doi:10.1088/0004-637X/746/2/142. arXiv: 1111.7186.

L. M. Oskinova, H. Todt, R. Ignace, J. C. Brown, J. P. Cassinelli, W.-R. Hamann, Early magnetic B-type stars: X-ray emission and wind properties, MNRAS 416 (2011) 1456-1474. doi:10.1111/j. 1365-2966.2011.19143.x. arXiv:1106.0508.

S. P. Owocki, A. ud-Doula, The effect of magnetic field tilt and divergence on the mass flux and flow speed in a line-driven stellar wind, ApJ 600 (2004) 1004-1015.

M. Palate, G. Rauw, Short-term spectroscopic variability of Plaskett's star, A\&A 572 (2014) A16. doi:10.1051/0004-6361/ 201423608. arXiv: 1410.1801.

E. Paunzen, M. Netopil, D. J. Bord, The first spectroscopic verification of an extragalactic classical chemically peculiar star, MNRAS 411 (2011) 260-262. doi:10.1111/j.1365-2966.2010.17682. x. arXiv: 1011.6162 .

V. Petit, D. Cohen, G. Wade, Y. Nazé, S. P. Owocki, J. O. Sundqvist, A. ud-Doula, A. W. Fullerton, M. A. Leutenegger, M. Gagné, Xray emission from the giant magnetosphere of the magnetic o-type star ngc 1624-2, MNRAS (2015) in press.

V. Petit, M. Gagné, D. H. Cohen, R. H. D. Townsend, M. A. Leutenegger, M. R. Savoy, G. Fehon, C. A. Cartagena, Solving the Puzzle of the Massive Star System $\theta^{2}$ Orionis A, in: A. C. Carciofi, T. Rivinius (Eds.), Circumstellar Dynamics at High Resolution, volume 464 of Astronomical Society of the Pacific Conference Series, 2012, p. 397. arXiv:1205.3538.

V. Petit, S. P. Owocki, G. A. Wade, D. H. Cohen, J. O. Sundqvist, M. Gagné, J. Maíz Apellániz, M. E. Oksala, D. A. Bohlender, T. Rivinius, H. F. Henrichs, E. Alecian, R. H. D. Townsend, A. udDoula, MiMeS Collaboration, A magnetic confinement versus rotation classification of massive-star magnetospheres, MNRAS 429 (2013) 398-422. doi:10.1093/mnras/sts344. arXiv: 1211.0282

J. Robrade, X-rays from A stars - Coronae and wind-shocks, in: G. Mathys, E. R. Griffin, O. Kochukhov, R. Monier, G. M. Wahlgren (Eds.), Putting A Stars into Context: Evolution, Environment, and Related Stars, 2014, pp. 425-434.

J. Robrade, J. H. M. M. Schmitt, New X-ray observations of IQ Aurigae and $\alpha^{2}$ Canum Venaticorum. Probing the magnetically channeled wind shock model in A0p stars, A\&A 531 (2011) A58. doi:10.1051/0004-6361/201116843. arXiv:1105.3688.

J. Sanz-Forcada, E. Franciosini, R. Pallavicini, XMM-Newton observations of the $\sigma$ Ori cluster. I. The complex RGS spectrum of the hot star $\sigma$ Ori AB, A\&A 421 (2004) 715-727.

N. S. Schulz, C. R. Canizares, D. Huenemoerder, J. C. Lee, X-Ray Line Emission from the Hot Stellar Wind of $\theta^{1}$ Orionis C, ApJ 545 (2000) L135-L139. doi:10.1086/317891. arXiv: astro-ph/0010310.

S. N. Shore, D. N. Brown, Magnetically controlled circumstellar matter in the helium-strong stars, ApJ 365 (1990) 665-676.

S. L. Skinner, K. R. Sokal, D. H. Cohen, M. Gagné, S. P. Owocki, R. D. Townsend, High-Resolution Chandra X-Ray Imaging and Spectroscopy of the $\sigma$ Orionis Cluster, ApJ 683 (2008) 796-812. doi:10.1086/589917. arXiv:0805.0714.

B. Stelzer, E. Flaccomio, T. Montmerle, G. Micela, S. Sciortino, F. Favata, T. Preibisch, E. D. Feigelson, X-Ray Emission from Early-Type Stars in the Orion Nebula Cluster, ApJS 160 (2005) 557-581. doi:10.1086/432375. arXiv: astro-ph/0505503.

B. Stelzer, C. A. Hummel, M. Schöller, S. Hubrig, C. Cowley, Search of X-ray emission from roAp stars: the case of $\gamma \mathrm{Equ}-$ ulei, A\&A 529 (2011) A29. doi:10.1051/0004-6361/201016265. arXiv: 1103.0739

J. O. Sundqvist, A. ud-Doula, S. P. Owocki, R. H. D. Townsend, I. D. Howarth, G. A. Wade, A dynamical magnetosphere model for periodic $\mathrm{H} \alpha$ emission from the slowly rotating magnetic O star HD 191612, MNRAS 423 (2012) L21-L25. doi:10.1111/j. 1745-3933.2012.01248.x. arXiv:1203.1050.

R. H. D. Townsend, S. P. Owocki, A rigidly rotating magnetosphere model for circumstellar emission from magnetic OB stars, MNRAS 357 (2005) 251-264. doi:10.1111/j.1365-2966.2005.08642. x. arXiv: astro-ph/0408565.

R. H. D. Townsend, S. P. Owocki, A rigidly rotating magnetosphere model for circumstellar emission from magnetic OB stars, MNRAS 357 (2005) 251-264. doi:10.1111/j.1365-2966.2005.08642.

R. H. D. Townsend, S. P. Owocki, A. ud-Doula, A Rigid-Field Hydrodynamics approach to modeling the magnetospheres of massive stars, MNRAS, in press 709 (2007). arXiv:0709.0694.

R. H. D. Townsend, T. Rivinius, J. F. Rowe, A. F. J. Moffat, J. M. Matthews, D. Bohlender, C. Neiner, J. H. Telting, D. B. Guenther, T. Kallinger, R. Kuschnig, S. M. Rucinski, D. Sasselov, W. W. Weiss, MOST Observations of $\sigma$ Ori E: Challenging the Centrifugal Breakout Narrative, ApJ 769 (2013) 33. doi:10.1088/0004-637X/769/1/33. arXiv:1304.2392.

A. ud-Doula, The effects of magnetic fields and field-aligned rotation on line-driven hot-star winds, Ph.D. thesis, University of Delaware, 2003.

A. ud-Doula, S. Owocki, R. Townsend, V. Petit, D. Cohen, Xrays from magnetically confined wind shocks: effect of coolingregulated shock retreat, MNRAS 441 (2014) 3600-3614. doi:10. 1093/mnras/stu769. arXiv: 1404.5336.

A. ud-Doula, S. P. Owocki, Dynamical simulations of magnetically channeled line-driven stellar winds. I. Isothermal, nonrotating, radially driven flow, ApJ 576 (2002) 413-428.

A. ud-Doula, S. P. Owocki, R. H. D. Townsend, Dynamical simulations of magnetically channelled line-driven stellar winds - II. The effects of field-aligned rotation, MNRAS 385 (2008) 97-108. doi:10.1111/j.1365-2966.2008.12840.x. arXiv:arXiv:0712.2780.

A. ud-Doula, S. P. Owocki, R. H. D. Townsend, Dynamical simulations of magnetically channelled line-driven stellar winds III. Angular momentum loss and rotational spin-down, MNRAS 392 (2009) 1022-1033. doi:10.1111/j.1365-2966.2008.14134.x. arXiv : 0810.4247

A. ud-Doula, J. O. Sundqvist, S. P. Owocki, V. Petit, R. H. D. Townsend, First 3DMHD simulation of a massive-star magnetosphere with application to $\mathrm{H} \alpha$ emission from $\theta^{1}$ Ori C, MNRAS 428 (2013) 2723-2730. doi:10.1093/mnras/sts246. arXiv: 1210.5298.

A. ud-Doula, J. O. Sundqvist, S. P. Owocki, V. Petit, R. H. D. Townsend, First 3DMHD simulation of a massive-star magnetosphere with application to $\mathrm{H} \alpha$ emission from $\theta^{1}$ Ori C, MNRAS 428 (2013) 2723-2730. doi:10.1093/mnras/sts 246. arXiv: 1210.5298

A. ud-Doula, R. H. D. Townsend, S. P. Owocki, Centrifugal Breakout of Magnetically Confined Line-driven Stellar Winds, ApJ 640 (2006) L191-L194. doi:10.1086/503382. arXiv:arXiv:astro-ph/0601193.

G. A. Wade, J. H. Grunhut, MiMeS Collaboration, The MiMeS Survey of Magnetism in Massive Stars, in: A. C. Carciofi, T. Rivinius (Eds.), Circumstellar Dynamics at High Resolution, volume 464 of Astronomical Society of the Pacific Conference Series, 2012, p. 405. arXiv: 1206.5163.

N. R. Walborn, N. I. Morrell, Y. Naze, G. A. Wade, S. Bagnulo, R. H. Barba, J. Maiz Apellaniz, I. D. Howarth, C. J. Evans, A. Sota, Spectral Variations of Of?p Oblique Magnetic Rotator Candidates in the Magellanic Clouds, ArXiv e-prints (2015). arXiv: 1507.02434.

E. J. Weber, L. J. Davis, The angular momentum of the Solar wind, ApJ 148 (1967) 217. 


\begin{abstract}
Reviewer number 1
"Magnetically Confined Wind Shocks in X-rays - a Review"

by ud-Doula and Naze gives an overview of the theoretical picture and the state of X-ray observations of massive stars with strong large-scale magnetic fields. They first present the theoretical perspective, beginning with the analytical model by Babel and Montmerle (1997) that first introduced the concept of MCWS in the context of Ap/Bp stars. They then present the results of the extensive simulation work on magnetic OB stars (where the winds are stronger and confinement weaker), beginning with ud-Doula and Owocki (2002), discuss the effects of shock retreat and stellar rotation, and relate the simulation results to the current state of X-ray observations. They then present the observational perspective. In that context they discuss the dependence of the X-ray luminosity on mass loss rate, how the magnetic field affects the spectrum, variability (both intrinsic and rotational), and what can be inferred about the distribution of the emitting gas from high-resolution spectra.

The final section lays out the prospect for future work in light of the upcoming Athenat mission and recent progress in theoretical work with the first 3D simulations and the development of the Arbitrary Rigid Field Hydrodynamics, which allows more realistic modelling of gas flows confined by very strong magnetic fields.
\end{abstract}

As this is a review article, the question of originality does not apply. The list of references is extensive, the language is clear, the figures in good quality, and the paper overall well-structured. I recommend publication without revision except for one correction: the beta parameter is mentioned in the text on page 2 (right column), but not included in the respective equations (left column). $>$ Done

Reviewer Number 2

The article is generally well written, concise and reflects the current state of the field.

A couple of minor corrections are requested, mostly dealing with syntax and structure issues or ambiguities in the text.

p1, 154, r: spelling inconsistency Ap-Bp vs. Ap/Bp (e.g. p3,11,1) $>$ Done

p1, 132, r : you define BM97a,b, but don't use it uniformly (e.g. p1, $155, r)$

$>$ Corrected

p1, 155, r: ....introduced XXX MCWS model. - word missing >Inserted 'the'.

Sect.2 The X-rays from IQ Aur in BM97a are predominantly very soft $(0.3$ $\mathrm{keV})$, not hard as suggested (e.g. p2, 14, 1)

The temperature/hardness depends on wind/shock speed, if these is quite low also MCWS can produce soft emission.

Should be clarified in the text, T_x Vs V relation could be included.

(Note: Significant hard X-ray emiss̄ion for IQ Aur was first seen by XMM, Robrade+Schmitt, 2011) 
>The purpose is to introduce the MCWS paradigm, but the softness of X-ray can be tied to 'shock retreat' described later. A sentence has been added.

p2, 119, 1: ...dominance of the field is determined how strong... syntax

Corrected: added 'by' in front of 'how'.

Sect.2.1 You state: for 'typical values...significant magnetic confinement...require fields of order \# $100 \mathrm{G} . '$

Assuming the 'typical values are 1 , is thus eta=0.4 considered as

'significant' confinement?

A few lines down you state eta=40 is 'substantial' and later eta=10^ 6 is

'extreme' or eta ge 0.1 is 'moderately small'

Are these more than linguistic terms?

Originally eta $<<1$ is 'weak' and eta $>>1$ is 'strong'; likewise also adapted here e.g. when simplifying eqn.4.

I always thought of a smooth transition from wind to magnetic field dominated regime.

Are there any threshold effects or phenomena that occur/dominate at a specific eta or eta-range?

If yes, please describe.

$>$ Confinement can occur only for eta>1, the degree of confinement will

vary how large eta is compared to 1 . Fields of order 100G, implies it could be $300 \mathrm{G}$ or even $400 \mathrm{G}$ but not $2 \mathrm{kG}$, on those cases indeed eta $\sim 1$, and thus there will be significant confinement. A sentence has been added to clarify this.

p2, 121, r: ...of magnetic XXX is set... - word missing

$>$ added 'confinement'

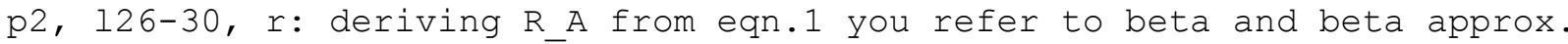
1 .

Clarify, what is beta and why it is 1 ? more confusing, there is no beta in eqn.1

$>$ it was an oversight, Going 2 nd equality to the 3 rd equality in eqn. there was an implicit assumption $\mathrm{v}^{\circledR}=\mathrm{v}_{\text {_inf }}\left(1-\mathrm{R}_{-} * / \mathrm{r}\right) * *$ beta. Typically, beta 0.8 for O-stars. For simplicity, more often than not, beta is assumed to be 1 . A sentence has been added to clarify.

p3, 141/42, I: as above, X-ray production does not solely depend on eta. Intrinsic wind speed matters.

$>$ Wind will be affected by the field and shock retreat that may

ultimately lower the shock speed. Therefore, we prefer to keep the text as is.

Sect 2.1.3: check semantics/syntax - reads like text copied out of context (e.g. what simplification has been relaxed?)

Not sure if you need an extra subsection here, fits to sect.3.

$>$ Agreed, and a few words have been added to clarify the simplification

(isothermal equation of state).

p3, 153, r: Similar study examining X-rays... - syntax/word missing

$>$ added 'predicted by'.

p4, 135, r: High value of... - syntax/word missing

>added a word. 
p5, 123, 1: ...massive stars' generally rapid rotation...

Aren't especially the magnetic ones comparable rather slow rotators?

later (p5, 135, r) you state: ...all known strongly magnetic o-stars are slow rotators...

Again, could be a linguistic issue, but might confuse the reader.

$>$ The first paragraph in section 2.3 has been modified to clarify this.

p5, 152, 1: What are the 'other B stars'? This is suggestive of magnetic

$B$ stars that are not Bp stars.

$>$ added other 'magnetic' B stars.

p5, 119, r: ....F(f)igure 4 where rapidly rotating B stars... No rotation is indicated in Fig.4, which are the rapid rotators here?

$>$ The referee is indeed right, we have deleted this reference to fig.4 and have simply referred the reader to sect 3 .

p5, 127-31, r: syntax corrupt/words missing; acronym WD defined, never used

$>$ added a few words and deleted the reference to WD.

p5, 134, r: ...effect on evolution of... - word missing

$>$ It was meant to be the evolution of these stars (discussed earlier)

General: While a linguistic break by a two-author review paper is hardly avoidable, you could tighten the connection between the modeling and observational part. e.g.p6,1 35, : ...scattered all over the so called 'magnetic confinement-rotation diagram' (Petit 2013) is actually the $\mathrm{CM} / \mathrm{DM}$ schema you describe in sect. 2.3.

$>$ It is in fact a stronger statement: not only do we have both CM and DM examples but e.g., amongst CMs we have different degrees of confinement and rotation (i.e. not all CMs are equal).

p6,133, r: ...the apparent hardness of spectrum... - word missing $>$ corrected

p6,137/38, r....simulations...is.. - grammar

$>$ corrected

p8, 119,1 : ...the soft character of the recorded $\mathrm{x}$-rays favor an origin of the flare in the Ap star...

The IQ Aur X-ray flare is bright, short and hard.

$>$ we refer here to sect 5.5 of Robrade \& Schmitt (2011): "However, young active stars have typically significantly higher plasma temperatures than observed for IQ Aur (see, e.g. Guedel 2004) and their X-ray light curves show quite frequent X-ray flares of factor up to a few, whereas IQ Aur is dominated by softer emission (Fig. 7) and exhibits only minor variability except for the large flare."

Words "(compared to young active stars)" have been added to clarify the text.

p8, 13-6, r: you bring up optical variability, I assume that the following paragraphs treat x-rays, please clarify

$>$ corrected

p9, 147-50, 1: You state that ... ra ring of negligible radius located at $1.15 R^{*}$ is dubious..., but why? 
Gagne 2005 find 1.2-1.5 $R^{*}$, not fundamentally different and they claim it fits to their $35 \%$ variation.

$>$ Please note that the MHD simulations predicted that "Most of the $\mathrm{X}$-rays are produced in a relatively small region around $R \sim 2 R * "$ (Gagne et al. 2005), i.e. close to the Alfven radius and note also that there was an erratum regarding He-like triplets (Gagne et al. 2005, ApJ, 634, 712713): the formation radii actually are 1.6-2 $\mathrm{R}^{*}$, not $1.2-1.5 \mathrm{R}^{*}$, and this new value agrees well with models but then it is not compatible with the small radius needed for the variations - as the authors mentioned "Thus, the only remaining discrepancy between the HETG data and the MCWS model is the overall X-ray variability, 1 - Lmin/Lmax $\sim 0.33$. If the X-rays are produced, on average, at $R \sim 2 R^{*}$, then occultation of an $\mathrm{X}$-ray torus by the photosphere would produce a $\sim 17 \%$ dip in visible $\mathrm{X}$-ray luminosity at phase 0.5 , only about half the observed drop." (text from erratum)

Direct reference to the erratum was provided, rather than to the initial paper, in sections 3.3 and 3.4 .

p10, 117, r: radii are typically measured from the core, but stating '...close to the photosphere, at 1-2R* from it...'

might suggest that you measure from the photosphere; clarify and check your numbers.

$>$ the quoted values were indeed counted from photosphere (i.e. $r-1 R^{*}$ ), they are now expressed in $r$ counted from core.

Overall, the second part of sect 3.3. would benefit from some structural revision.

Obviously you can produce all sorts of phases light curves by various configuration but

aspects like spectral variability or extreme variations remain

unexplained from Fig.5, so what is the conclusion here?

$>$ The conclusion as stated at the end of section 3.3 is that the shape of variations can be reproduced but not their amplitude - though usually people thinks that occultation of a ring would be sufficient. We also shortened sections 3.3 and 3.4 .

Also the organization of the paragraph is a bit confusing and topics mix, further there are several repetition,

e.g flux changes of Tr16-22 are mentioned at least three times.

$>$ the text presents the two possibilities, first flares than rotational

modulation but rotational modulation is vast : it includes non-dipolar

cases (tau sco) and dipolar cases (the other ones) and its explanation is either linked to absorption or geometry. We have considered these in turn. The text has been somewhat shortened, we've tried to improve this section. Note that allusions to Tr16-22 have been reduced.

A separation between flaring and rotational modulation into two subsections might also help.

$>$ done

p10, 146, r :...extremely low mass rate $B$ stars...- syntax/word missing > corrected

$>$ corrected 